Research Paper

\title{
Analysis of Clinical characteristics to predict pathologic complete response for patients with locally advanced rectal cancer treated with neoadjuvant chemoradiotherapy
}

\author{
Haihua Peng ${ }^{1,2^{*}}$, Chengtao Wang ${ }^{*}$, Weiwei Xiao ${ }^{3^{*}}$, Xiaodan Lin ${ }^{1}$, Kaiyun You ${ }^{4}$, Jun Dong2 ${ }^{2}$ Zhenyu Wang2, \\ Xiaobi Yu², Zhifan Zeng ${ }^{3}$, Tongchong Zhou1, Yuanhong Gao ${ }^{3 凶}$, Bixiu Wen ${ }^{2 \bowtie}$ \\ 1. Department of Radiation Oncology, Affiliated Cancer Hospital \& Institute of Guangzhou Medical University, Guangzhou 510075, China \\ 2. Department of Radiation Oncology, The First Affiliated Hospital of Sun Yat-sen University, Guangzhou 510080, China \\ 3. Department of Radiation Oncology, Sun Yat-Sen University Cancer Center, State Key Laboratory of Oncology in Southern China, Guangzhou 510060, China \\ 4. Department of Radiation Oncology, Sun Yat-sen Memorial Hospital, Sun Yat-sen University, Guangzhou 510120, China \\ *: these authors contributed equally to this work. \\ $\triangle$ Corresponding authors: 1. Bixiu Wen, M.D., Ph.D. Tel: +8620-87755766-6071; Email: wenbix@mail.sysu.edu.cn. 2. Yuanhong Gao, M.D., Ph.D. Tel: \\ +8620-87343385; Email: gaoyh@sysucc.org.cn \\ (c) Ivyspring International Publisher. This is an open access article distributed under the terms of the Creative Commons Attribution (CC BY-NC) license \\ (https://creativecommons.org/licenses/by-nc/4.0/). See http://ivyspring.com/terms for full terms and conditions.
}

Received: 2018.02.10; Accepted: 2018.04.27; Published: 2018.06.23

\begin{abstract}
To explore clinical characteristics which could be applied to predict pathologic complete response $(\mathrm{PCR})$ for patients with locally advanced rectal cancer treated with neoadjuvant chemoradiotherapy (neo-CRT) and total mesorectal excision (TME). 297 patients with locally advanced rectal cancer (cT3-4 or $\mathrm{CN}+$ ) who were treated with neo-CRT followed by TME were retrospectively reviewed. Clinical characteristics including age, gender, tumor distance from anus, serum CEA, hemoglobin levels before treatment and clinical TN stage were used to investigate the association with PCR after neo-CRT. Seventy-nine (26.6\%) patients achieved PCR after neo-CRT. PCR were achieved in 42 (34.4\%) patients in cTl-3 stage and 37 (21.1\%) in cT4 stage. PCR rate was $36.4 \%$ and $16.4 \%$ for patients with pre-treatment serum CEA $\leq 5.33 \mathrm{ng} / \mathrm{ml}$ and $>5.33 \mathrm{ng} / \mathrm{ml}$, respectively. Uni- and multi-variate analyses revealed that pre-treatment serum CEA level $\leq 5.33 \mathrm{ng} / \mathrm{ml}$ and clinical T stage, (i.e., cT1-3 versus cT4) were highly correlated with PCR $(\mathrm{p}<0.05)$. Clinical T stage and pre-treatment serum CEA level were strongly associated with $p C R$ for patients with locally advanced rectal cancer treated with neo-CRT followed by TME which could be applied as clinical predictors for PCR.
\end{abstract}

Key words: Rectal cancer; Neoadjuvant chemoradiotherapy; Total mesorectal excision; TN stage; CEA; Pathologic complete response

\section{Introduction}

Colorectal cancer is the $5^{\text {th }}$ most common cancer in morbidity and mortality in China according to Globocan 2012 [1, 2]; while approximately $40 \%$ of the patients would be diagnosed as rectal cancer. Among them $49.9 \%$ have been diagnosed as Duck B stage, $33.9 \%$ as Duck C [3]. Meta analysis of CONCORD-2 has been shown that the 5-year survival from colon and rectal cancers has increased steadily to $60 \%$ and higher in 22 developed countries especially for those diagnosed during 2005-09 [4].

Neoadjuvant chemoradiotherapy (neo-CRT) in combination with total mesorectal excision (TME) followed by adjuvant chemotherapy has been considered as the standard of care for patients with locally advanced rectal cancer. Clinical researches have been shown that the application of neo-CRT can 
shrink tumor, reduce local tumor recurrence, increase sphincter-perservation and further improve the prognosis and quality of life while not increase surgical complications and sequelae in this cohort of patients[5]; hence neo-CRT have been widely applied in the management of locally advanced rectal cancer.

It has been reported in the literature that 18.1 to 30.2 percent of patients with locally advanced rectal cancer may achieve pathologic complete response (pCR) after neo-CRT which leads to a better clinical prognosis [6-9]. Patients with locally advanced rectal cancer have been shown to present different treatment response to neo-CRT ranging from $\mathrm{pCR}$ to resistance to neo-CRT; whereas pathological TNM stage has been reported to be of prognostic significance in treatment outcomes after neo-CRT when compared to clinical TNM stage. Bujko et al. [10] have reported that prognostic effect of adjuvant chemotherapy in patients with ypT0-4 N0 disease after Neo-CRT and TME is not convincing, suggesting that adjuvant chemotherapy should be given individually according to pathological TNM stage. We have reported that the pCR rate was achieved in $24.8 \%$ of 210 patients who were demonstrated with better prognosis when compared with those with advanced pathologic $\mathrm{T}$ and $\mathrm{N}$ stage treated with neo-CRT and TME [6].

The purpose of the study was to explore clinical characteristics which could be applies to predict treatment response especially $\mathrm{pCR}$ to neo-CRT in patients with locally advanced rectal cancer who were treated with neo-CRT and TME.

\section{Methods}

297 patients with locally advanced rectal cancer were consecutively treated with neo-CRT followed by TME with or without adjuvant chemotherapy between March 2003 and December 2012. The treatment strategies were made according to rectum cancer National Comprehensive Cancer Network (NCCN) guidelines. All patients who were pathologically diagnosed as rectal cancer were performed clinical stage examinations including physical exam, serum chemical profile including carcinoembryonic antigen (CEA), colonoscopy and two to three image studies, i.e., chest radiography, barium enema, chest/abdomen/pelvic computed tomography (CT), pelvic magnetic resonance image (MRI), intraluminal ultrasound (ERUS), positron emission computed tomography (PET/CT) and emission computed tomography (ECT) according to American Joint of Cancer Classification (AJCC) 2002 [11].

Preoperative radiotherapy (RT) was performed with three-dimensional conformal radiation therapy
(3D-CRT) in 240 patients $(80.8 \%)$ and intensity modulated radiation therapy (IMRT) in 57 patients $(19.2 \%)$ with 6-8 MV X-ray. The target volume definition was followed by the recommendations of International Commission of Radiation Units report 50. The delineation of clinical target volume (CTV) included primary rectal cancer, both ends of the affected rectum, the surrounding tissues of the affected rectum, the mesorectal area, the presacral lymph nodes, the obtrurator lymph nodes, and the iliac lymph nodes. For patients with stage T4 rectal carcinoma with bladder or prostate involvement, the delineation of CTV also included external iliac lymph nodes.

The planned target volume (PTV) was designated as $5-10 \mathrm{~mm}$ margin from the CTV. The dose prescription was as follows: $100 \%$ of the prescription dose covered at least $95 \%$ volume of the PTV; 95\% of the prescription dose covered 100\% volume of the PTV. The reference point was set as the intersection of the central axes of the three or four beams for 3D-CRT or five beams for IMRT. The median radiation dose to PTV was 46.0 Gy (ranging from 30.0 to $50.0 \mathrm{~Gy}$ ), 2 Gy per fraction, 5 fractions per week. The dose to the OARs was aimed to be as low as possible and must at least comply with the following constraints: bladder of 50 Gy in $<50 \%$ volume; Dmean of small bowel < 46 Gy, small bowel of 50 Gy in $<5 \%$ volume.

Two regimens of chemotherapy were administered during radiotherapy either the FOLFOX (Fluorouracil, $3.0 \mathrm{~g} / \mathrm{m}^{2}$, IV continuous infusion for 48 $\mathrm{h}$ on day 1; Leucovorin calcium, $200.0 \mathrm{mg} / \mathrm{m}^{2}$, IV bolus on day 1 ; Oxaliplatin, $100.0 \mathrm{mg} / \mathrm{m}^{2}$, IV on day 1 ; two cycles at an interval of 3 weeks), or the XELOX (Capecitabine, $1000.0 \mathrm{mg} / \mathrm{m}^{2}$, on $\mathrm{d} 1-14$; Oxaliplatin, $100.0 \mathrm{mg} / \mathrm{m}^{2}$, IV on day 1; two cycles at an interval of 3 weeks). The postoperative adjuvant chemotherapy was either one of FOLFOX, XELOX or Capecitabine alone with median cycles of 3 (range from 2 to 6 cycles).

Radical surgery was performed according to the principles of total mesorectal excision at approximately 45 days (range: 20 -142 days) after completion of neo-CRT. Postoperative pathological examination was performed according to the criteria developed by the AJCC/UICC (2002).

Treatment response including tumor regression response to neo-CRT was investigated according to the clinical characteristics including age, gender, tumor distance from anus, serum CEA, hemoglobin levels before treatment and clinical TN stage were used to investigate association with $\mathrm{pCR}$ after neo-CRT. 
All statistical analyses were performed using SPSS v17.0 software. The receiver-operating characteristic curve (the ROC curve) was applied to obtain a boundary value of pre-serum CEA to determine the correlation to $\mathrm{pCR}$. Chi-square test was utilized to analyze clinical factors associated with pCR. Logistic regression was applied to analyze clinical factors associated with pCR. Uni- and multi-variate analyses of the prognostic factors were performed using Cox proportional hazard models. $\mathrm{p}$ value of $<0.05$ was considered to be of statistical significance.

Table 1. The patient characteristics for 297 patients with locally advanced rectum cancer patients

\begin{tabular}{|c|c|c|}
\hline Clinical factors & & $\%$ \\
\hline \multicolumn{3}{|l|}{ Gender (number) } \\
\hline Male & 207 & 69.7 \\
\hline Female & 90 & 30.3 \\
\hline Median age (year, range) & $56(15-80)$ & \\
\hline Median Pre-CEA level (ng/ml, range) & $4.5(0.2-249.6)$ & \\
\hline Median Pre- $\mathrm{Hb}(\mathrm{g} / \mathrm{L} \text {, range })^{\$}$ & $130(64-170)$ & \\
\hline Distance anal verge (cm, range) & $5(1-15)$ & \\
\hline \multicolumn{3}{|l|}{ Methods clinical stage (number) } \\
\hline ERUS & 274 & 92.3 \\
\hline $\mathrm{CT}$ & 189 & 63.6 \\
\hline MRI & 87 & 29.3 \\
\hline \multicolumn{3}{|l|}{ Clinical T (number) } \\
\hline $\mathrm{T} 1$ & 4 & 1.3 \\
\hline $\mathrm{T} 2$ & 8 & 2.7 \\
\hline $\mathrm{T} 3$ & 110 & 37 \\
\hline $\mathrm{T} 4$ & 175 & 58.9 \\
\hline \multicolumn{3}{|l|}{ Clinical N (number) } \\
\hline No & 92 & 31.0 \\
\hline $\mathrm{N}+$ & 205 & 69.0 \\
\hline \multicolumn{3}{|l|}{ Histologic grade (number) } \\
\hline G1 & 21 & 7.1 \\
\hline G2 & 238 & 80.1 \\
\hline G3 & 38 & 12.8 \\
\hline Median preoperative radiotherapy dose (Gy, range) & $46(30-50)$ & \\
\hline \multicolumn{3}{|l|}{ Prechemotherapy (number) } \\
\hline FOLFOX & 47 & 16.1 \\
\hline XELOX & 229 & 78.4 \\
\hline others & 16 & 5.5 \\
\hline $\begin{array}{l}\text { Interval between completion of neo-CRT and surgery } \\
\text { (day, range) }\end{array}$ & $45(20-142)$ & \\
\hline \multicolumn{3}{|l|}{ Surgery (number) } \\
\hline Miles & 129 & 43.4 \\
\hline Dixon & 159 & 53.5 \\
\hline Hartmann & 9 & 3 \\
\hline Median lymph node from surgery (number, range) & $5(0-27)$ & \\
\hline
\end{tabular}

\$: Pre-Hb: pre-treatment level of hemoglobin

\section{Results}

\section{Patient characteristics}

There were totally 297 patients including 207 males (69.7\%) and 90 females (30.3\%) with median age of 56 years ranging from 15 to 80 years. The median CEA level was $4.5 \mathrm{ng} / \mathrm{ml}$ ranging from 0.2 to 249.6 $\mathrm{ng} / \mathrm{ml}$. The median tumor location of low-lying (distance between anal verge and lower edge of tumor) was $5 \mathrm{~cm}$. Patients with clinical stage II and III were $90(30.3 \%)$ and $207(69.7 \%)$, respectively. The detailed patient characteristics are shown in Table 1.
The total mesorectal excision was performed at 45 days (range: 20-142 days) after completion of neo-CRT. All patients were operated on R0 resection with Dixon procedure in 159 patients (53.5\%), Miles procedure in 129 patients $(43.4 \%)$ and Hartmann procedure in 9 patients $(3.0 \%)$. The detailed surgical procedures are also shown in Table 1.

\section{Evaluation of treatment response to neo-CRT}

Postoperative pathological evaluation after neo-CRT according to TNM classification was made on the samples from total mesorectal excision. Among them, ypT0N0 (pathologic complete response, pCR) was observed in 79 patients $(26.6 \%)$, while ypT1-2N0 in 57 patients $(19.2 \%)$, ypT3-4N0 in $94(31.6 \%)$ and ypT0-4N+ in $67(22.6 \%)$ patients, respectively.

\section{Correlation of the baseline CEA level with PCR after neo-CRT}

The cut-off of pre-treatment CEA level to predict pCR was $5.33 \mathrm{ng} / \mathrm{ml}$ using ROC curve calculation. Area under the ROC curve was 0.675 (0.608 to 0.742 ), prompted use pre-treatment CEA to predict the $\mathrm{pCR}$ has certain accuracy. As shown in Fig. 1, the probability to achieve $\mathrm{pCR}$ after neo-CRT for patients with baseline level of CEA $<5.33 \mathrm{ng} / \mathrm{ml}$ or $\geq 5.33$ $\mathrm{ng} / \mathrm{ml}$ was $36.5 \%$ and $13.8 \%$, respectively, in which the difference is statistically significant.

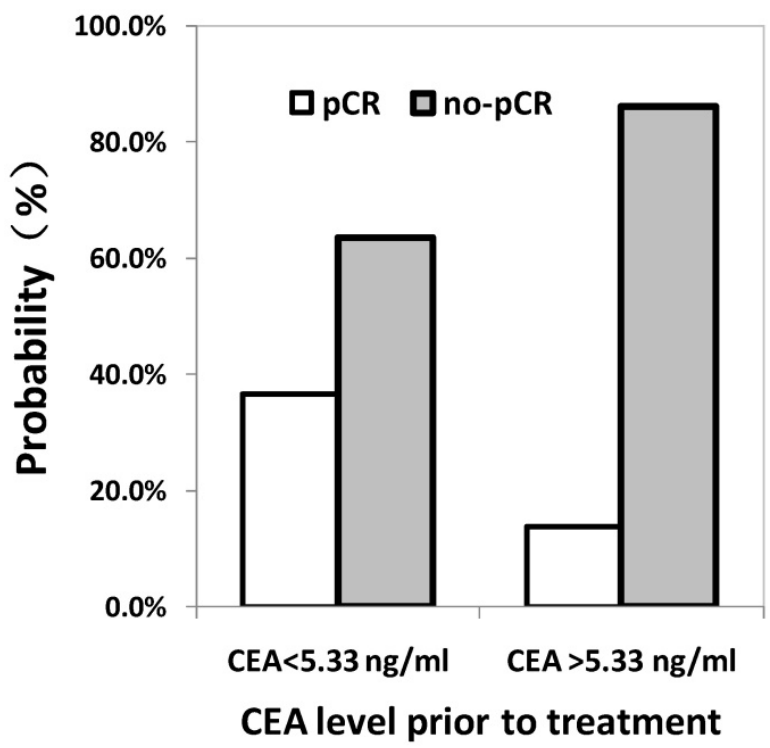

Figure 1. The correlation between pathologic complete response $(p C R)$ and pre-CEA level determined by the ROC curve.

\section{Uni- and multi-variate logistic analyses of clinical predictors for $\mathbf{P C R}$}

Univariate analysis showed that patients with clinical T1-3 ( $p=0.012)$ and pre-treatment CEA level less than $5.33 \mathrm{ng} / \mathrm{ml}(\mathrm{p}<0.001)$ were associated with higher possibility to achieve pCR after neo-CRT (Fig. 
2). As shown in Table 2, gender, age, pre-treatment level of hemoglobin, distance between the anal verge and the lower tumor edge, pathological differentiation, clinical $\mathrm{N}$ stage, interval between completion of neo-CRT and total mesorectal excision were not associated with $\mathrm{PCR}$.

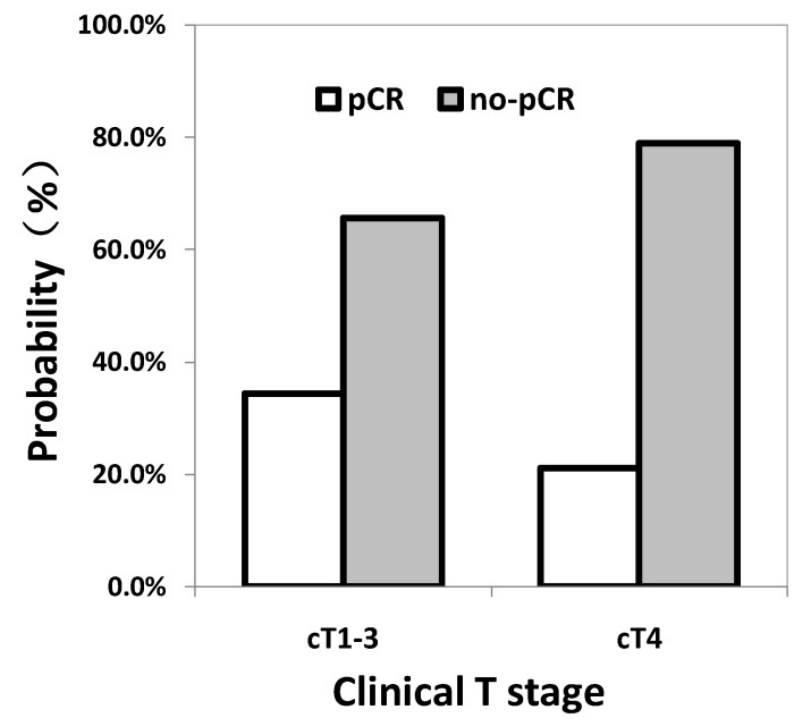

Figure 2. The relationship between the pathologic complete response (PCR) and the clinical T stage.

Table 2. Univariate logistic analyses of predictors for $P C R$

\begin{tabular}{|c|c|c|c|}
\hline Variable & Number & pCR N (\%) & $\mathrm{p}$ value \\
\hline Gender & & & 0.986 \\
\hline Male & 207 & $55(26.6)$ & \\
\hline Female & 90 & $24(26.7)$ & \\
\hline Age ${ }^{\star}$ & & & 0.163 \\
\hline$\leq 56$ & 159 & $37(23.3)$ & \\
\hline$>56$ & 138 & $42(30.4)$ & \\
\hline Pre-Hb ${ }^{\circledast}(g / L)$ & & & 0.348 \\
\hline Anemia & 55 & $12(21.8)$ & \\
\hline Normal & 228 & $64(28.1)$ & \\
\hline Pre-CEA (ng/mL) & & & $<0.001$ \\
\hline$\leq 5.33$ & 167 & $61(36.5)$ & \\
\hline$>5.33$ & 130 & $18(13.8)$ & \\
\hline \multicolumn{3}{|c|}{ Distance between lower border of tumor and anal verge $(\mathrm{cm})$} & 0.464 \\
\hline$\leq 5$ & 172 & $43(25.0)$ & \\
\hline$>5$ & 125 & $36(28.8)$ & \\
\hline Histological grade & & & 0.74 \\
\hline G1 & 21 & $5(23.8)$ & \\
\hline G2 & 238 & $62(26.1)$ & \\
\hline G3 & 38 & $12(31.6)$ & \\
\hline Clinical T & & & 0.011 \\
\hline T1-3 & 122 & $42(34.4)$ & \\
\hline T4 & 175 & $37(21.1)$ & \\
\hline Clinical N & & & 0.886 \\
\hline No & 92 & $23(25.0)$ & \\
\hline $\mathrm{N}+$ & 205 & $56(27.3)$ & \\
\hline Clinical stage & & & 0.579 \\
\hline II & 90 & $22(24.4)$ & \\
\hline III & 207 & $57(27.5)$ & \\
\hline \multicolumn{3}{|c|}{ Interval between completion neo-CRTand surgery (week) } & 0.658 \\
\hline$\leq 4$ & 8 & $1(12.5 \%)$ & \\
\hline $4 \sim 8$ & 237 & $64(27.0 \%)$ & \\
\hline$\geq 8$ & 52 & $14(26.9 \%)$ & \\
\hline
\end{tabular}

@: grouped by whether anemia, male normal $120 \mathrm{~g} / \mathrm{L}$, female normal $110 \mathrm{~g} / \mathrm{L}$; \&: grouped by median
Table 3. Multivariate logistic analyses of predictors for $\mathrm{PCR}$

\begin{tabular}{lll}
\hline Variable & HR $(\mathbf{9 5} \% \mathrm{CI})$ & p value \\
\hline CEA $(\mathrm{CEA} \leq 5.33 \mathrm{ng} / \mathrm{ml}$ vs. CEA $>5.33 \mathrm{ng} / \mathrm{ml})$ & $3.434(1.897-6.215)$ & $<0.001$ \\
Clinical T stage $(\mathrm{cT} 1-3$ vs.cT4) & $1.808(1.056-3.095$ & 0.031 \\
\hline HR. hazard ratio. CI. confidence interval & &
\end{tabular}

As depicted in Table 3, multivariate analyses showed that pre-treatment CEA level, clinical T1-3 were associated with $\mathrm{PCR}$.

\section{Discussion}

The present study has been shown that $26.6 \%$ of the patients with locally advanced rectal cancer have achieved pathologic complete response when they were treated with neo-CRT followed by TME. Pre-treatment serum CEA level and clinical $\mathrm{T}$ stage were strongly associated with $\mathrm{pCR}$ for this cohort of patients treated with neo-CRT followed by TME which could be used as clinical predictors for $\mathrm{pCR}$.

Recent studies have been reported that postoperative pathological stage after neo-CRT was strongly correlated with prognosis for patients with locally advanced rectal cancer. Quah et al [12] have demonstrated that postoperative stage (ypT0N0, ypT1-2N0, ypT3-4N0 and ypN+) in 331 patients was significantly associated with disease free survival $(p=0.003)$, and overall survival $(p=0.000)$, especially for those who were achieved complete or near complete pathologic response. Park et al [7] have reported in a cohort of 725 patients with locally advanced rectal cancer that 5 year overall survival for those with stage ypT0N0, ypT1-2N0, and ypT3-4/N+ rectal was $93.4 \%, 87.0 \%$, and $77.3 \%$, respectively $(\mathrm{p}=0.002)$; the 5 year DFS was $90.5 \%, 78.7 \%$, and 58.5 $\%$, respectively $(\mathrm{p}<0.001)$.

Given that the postoperative pathological stage after neoadjuvant chemoradiotherapy was an important predictor for prognosis, postoperative adjuvant therapeutics would be recommended according to the response of rectal cancer to neo-CRT. We and other researchers [13-15] have demonstrated that pathological TN stage was strongly associated with treatment outcome for those who were treated with neoadjuvant chemoradiotherapy followed by total mesorectal excision, especially for those with pCR after neo-CRT could achieve a better prognosis. For example, a wait-and-see strategy could be applied in patients with stage ypTON0 rectal cancer [16]; intensified adjuvant chemotherapy could be applied in patients with stage ypT3-4/N+ colorectal cancer. Whereas the ability to monitor $\mathrm{pCR}$ patients after neo-CRT before radical surgery would significantly impact subsequent management. Patients who would achieve PCR after neo-CRT may be recommended to have local excision or wait-and-see treatment strategy 
to avoid radical surgery-related sequelae and complications.

It remains controversial how to screen patients who were going to benefit from neo-CRT and achieve pCR for those with locally advanced rectal cancer. CEA is a glycoprotein secreted by colorectal cancer tissue, which have been widely detected in the origin of the germ layer within the digestive system tumors; it can also exist in normal human serum but remains at very low level. The detection of CEA has extensively been used in clinic to screen patients with colorectal cancer, monitor treatment response and predict prognosis due to its convenience, technique maturity and low cost. There have been reports on the research of relationship between the CEA level and prognosis for patients with colorectal cancer. Searcher $[17,18]$ reported concentrations of serum CEA was significantly related to the overall survival and recurrence rate for patients with colorectal cancer.

Das et al [19] have reported in 562 patients with locally advanced rectal cancer that those with the CEA $\leq 2.5 \mathrm{ng} / \mathrm{mL}$ was associated with higher $\mathrm{pCR}$ rate after neo-CRT than those with CEA $>2.5 \mathrm{ng} / \mathrm{mL}$ ( $24 \%$ vs $11 \%, \mathrm{p}=0.015)$. Moureau-Zabotto and Steinhagen et al $[20,21]$ have made similar report with a cutoff of pre-treatment CEA level of $5 \mathrm{ng} / \mathrm{ml}$ or $2.5 \mathrm{ng} / \mathrm{ml}$ in patients with locally advanced rectal cancer patients.

The pre-treatment CEA level has been shown certain correlation with $\mathrm{pCR}$, whereas there is still no suitable standard to select the cut-off level of the CEA that could be used to predict $\mathrm{pCR}$ to neo-CRT. The present study has applied ROC curve to screen the most optimal CEA cutoff value $(5.33 \mathrm{ng} / \mathrm{ml})$ to investigate the relationship between the pre-treament CEA level and pCR in response to neo-CRT. Our study suggested that patient with pre-treatment CEA level $\leq 5.33 \mathrm{ng} / \mathrm{ml}$ may have higher chance to achieve pCR after neo-CRT than those with pre-treatment CEA level of $>5.33 \mathrm{ng} / \mathrm{ml}$ (36.5\% vs $13.8 \%)$.

Rodel et al [22] have reported that 4 out of 16 patient $(25 \%)$ with clinical T2 rectal cancer achieved pCR after neo-CRT, while 27 out of 268 patients (10.1\%) with clinical T3 disease achieved pCR and none of 27 patients with $\mathrm{T} 4$ disease achieved $\mathrm{pCR}$. The authors have postulated that the tumor volume was related to its sensitivity to radiation and chemotherapy, and that there might exist a certain correlation between tumor volume and clinical stage. The present study has shown that patients with clinical T1-3 rectal cancer would have higher probability to achieve $\mathrm{pCR}$ after neo-CRT than those with $\mathrm{T} 4$ disease $(34.4 \%$ vs $21.1 \%)$. We may hypothesize that higher $\mathrm{T}$ staging prior treatment meant heavier tumor burden, while the higher radiation dose or more intense chemotherapy may be needed to achieve a better curative effect.

Due to relative small sample size, we combined $\mathrm{T} 1, \mathrm{~T} 2$ and T3 altogether to investigate the relationship between $\mathrm{T}$ stage and $\mathrm{pCR}$ to neo-CRT. Uni- and multi-variate analyses have demonstrated that clinical $\mathrm{T}$ stage and pre-treatment CEA leve $\leq 5.33$ $\mathrm{ng} / \mathrm{ml}$ were significantly related to $\mathrm{pCR}$ after neo-CRT for patients with locally advanced rectal cancer. It is important to design a prospective clinical trial to further study whether clinical $\mathrm{T}$ stage and pre-treatment CEA level are correlated with $\mathrm{PCR}$ to neo-CRT in patients with locally advanced rectal cancer.

In conclusion, our research has demonstrated that clinical stage and pre-treatment CEA level before treatment are independent predictive factors for patients with locally advanced rectal cancer to achieve pathologic complete response after neo-CRT followed by total mesorectal excision. The role of pre-treatment serum CEA level and clinical $\mathrm{T}$ stage to predict pathologic complete response to neo-CRT is needed for further evaluation.

\section{Abbreviations}

pCR: pathologic complete response; neo-CRT: neoadjuvant chemoradiotherapy; TME: total mesorectal excision; neo-CRT: Neoadjuvant chemoradiotherapy; NCCN: National Comprehensive Cancer Network NCCN; CEA: carcinoembryonic antigen; CT: computed tomography; MRI: magnetic resonance image; ERUS: intraluminal ultrasound; PET/CT: positron emission computed tomography; ECT: emission computed tomography; AJCC: American Joint of Cancer Classification; RT: radiotherapy; IMRT: intensity modulated radiation therapy; CTV: clinical target volume; PTV: planned target volume.

\section{Acknowledgements}

This work was partly funded by National Natural Science Foundation of China (No. 81673088).

\section{Author Contributions}

BW, YG designed the study. CW, WX, XL, KY, $\mathrm{JD}, \mathrm{ZW}, \mathrm{XY}, \mathrm{TZ}, \mathrm{ZZ}$ participated in the data collection and data analyses. HP, YG, BW wrote the manuscript. All authors read and approved the final manuscript.

\section{Competing Interests}

The authors have declared that no competing interest exists.

\section{References}

1. Torre LA, Bray F, Siegel RL, et al. Global cancer statistics, 2012. CA Cancer J Clin. 2015; 65: 87-108. 
2. Chen W, Zheng R, Baade PD, et al. Cancer statistics in China, 2015. CA Cancer J Clin. 2016; 66: 115-32.

3. Cheng L, Eng C, Nieman LZ, et al. Trends in colorectal cancer incidence by anatomic site and disease stage in the United States from 1976 to 2005. Am J Clin Oncol. 2011; 34: 573-80.

4. Allemani C, Weir HK., Carreira H, et al. Global surveillance of cancer survival 1995-2009: analysis of individual data for 25,676,887 patients from 279 population-based registries in 67 countries (CONCORD-2). Lancet. 2015;385: 977-1010.

5. Sauer R, Becker H, Hohenberger W, et al. Preoperative versus postoperative chemoradiotherapy for rectal cancer. N Engl J Med. 2004;351: 1731-40.

6. Wen B, Zhang L, Wang C, et al. Prognostic significance of clinical and pathological stages on locally advanced rectal carcinoma after neoadjuvant chemoradiotherapy. Radiat Oncol. 2015; 10: 124.

7. Park IJ, You YN, Agarwal A, et al. Neoadjuvant treatment response as an early response indicator for patients with rectal cancer. J Clin Oncol. 2012;30: 1770-6.

8. de Campos-Lobato LF, Stocchi L, da Luz Moreira A, et al. Pathologic complete response after neoadjuvant treatment for rectal cancer decreases distant recurrence and could eradicate local recurrence. Ann Surg Oncol. 2011;18: 1590-8.

9. Stipa F, Chessin DB, Shia J, et al. A pathologic complete response of rectal cancer to preoperative combined-modality therapy results in improved oncological outcome compared with those who achieve no downstaging on the basis of preoperative endorectal ultrasonography. Ann Surg Oncol. 2006;13: 1047-53.

10. Bujko K, Glynne-Jones R, Bujko M. Does adjuvant fluoropyrimidine-based chemotherapy provide a benefit for patients with resected rectal cancer who have already received neoadjuvant radiochemotherapy? A systematic review of randomised trials. Ann Oncol. 2010;21: 1743-50.

11. Greene FL, Page DL, Fleming ID, et al. AJCC cancer staging handbook, 6th ed. New York, NY: Springer Verlag, 2002 : 113-123

12. Quah HM, Chou JF, Gonen M, et al. Pathologic stage is most prognostic of disease-free survival in locally advanced rectal cancer patients after preoperative chemoradiation. Cancer. 2008;113: 57-64.

13. Kundel $\mathrm{Y}$, Brenner $\mathrm{R}$, Purim $\mathrm{O}$, et al. Is local excision after complete pathological response to neoadjuvant chemoradiation for rectal cancer an acceptable treatment option? Dis Colon Rectum. 2010; 53: 1624-31.

14. Callender GG, Das P, Rodriguez-Bigas MA, et al. Local excision after preoperative chemoradiation results in an equivalent outcome to total mesorectal excision in selected patients with T3 rectal cancer. Ann Surg Oncol. 2010; 17: 441-7.

15. Belluco C, De Paoli A, Canzonieri V, et al. Long-term outcome of patients with complete pathologic response after neoadjuvant chemoradiation for CT3 rectal cancer: implications for local excision surgical strategies. Ann Surg Oncol. 2011; 18: 3686-93.

16. Wolthuis AM, Penninckx F, Haustermans K., et al. Outcome standards for an organ preservation strategy in stage II and III rectal adenocarcinoma after neoadjuvant chemoradiation. Ann Surg Oncol. 2011;18: 684-90.

17. Tarantino I, Warschkow R, Worni M, et al. Elevated preoperative CEA is associated with worse survival in stage I-III rectal cancer patients. Br J Cancer. 2012;107: 266-74

18. Song S, Hong JC, McDonnell SE, et al. Combined modality therapy for rectal cancer: the relative value of posttreatment versus pre-treatment CEA as a prognostic marker for disease recurrence. Ann Surg Oncol. 2012;19: 2471-6.

19. Das P, Skibber JM, Rodriguez-Bigas M.A, et al. Predictors of tumor response and downstaging in patients who receive preoperative chemoradiation for rectal cancer. Cancer. 2007;109: 1750-5.

20. Moureau-Zabotto L, Farnault B, de Chaisemartin C, et al. Predictive factors of tumor response after neoadjuvant chemoradiation for locally advanced rectal cancer. Int J Radiat Oncol Biol Phys. 2011; 80: 483-91.

21. Steinhagen E, Shia J, Riedel E, et al. Response to neoadjuvant therapy in patients with early age-of-onset rectal cancer. Dis Colon Rectum.2013; 56: 58-63.

22. Rodel C, Martus P, Papadoupolos T, et al. Prognostic significance of tumor regression after preoperative chemoradiotherapy for rectal cancer. J Clin Oncol.2005; 23: 8688-96. 